\title{
Theoretical evaluation of equilibrium constant for boron isotopes exchange between boric acid and borate in pure and saline water
}

\author{
Amin Alibakhshi ${ }^{1, *}$, Julien Steffen ${ }^{1}$, Carlos Pinilla ${ }^{2,3}$, Bernd Hartke ${ }^{1}$ \\ ${ }^{1}$ Theoretical Chemistry, Institute for Physical Chemistry, Christian-Albrechts-University, Olshausenstr. \\ 40, 24118 Kiel, Germany \\ ${ }^{2}$ Departamento de Física y Geociencias, Universidad del Norte, Km 5 via Puerto Colombia, Colombia \\ ${ }^{3}$ School of Chemistry, University of Bristol, Cantock's Close Road, BS8 1TS, Bristol, United Kingdom \\ Corresponding author: alibakhshi@pctc.uni-kiel.de
}

\begin{abstract}
Evaluation of the equilibrium constant of boron isotope fractionation between boric acid and borate $\left(\mathrm{k}_{3-4}\right)$ in water is of high geochemical importance, due to its contribution in reconstruction of ancient seawater $\mathrm{pH}$ and atmospheric $\mathrm{CO}_{2}$. As a result, precise evaluation of $\mathrm{k}_{3-4}$ has been the subject of numerous studies, yielding diverse and controversial results. In the present study, employing three different rigorous and high-precision theoretical approaches, we provide a reliable estimation of $\mathrm{k}_{3-4}$ which is a value between 1.028 to 1.030 for both pure and saline water. Within the context of present study, we also propose partial normal mode analysis, Boltzmann weighted averaging and a revision on the Bigeleisen and Mayer method which allow a more rigorous evaluation of isotope fraction in solution and can be used for studying other isotopic systems as well.
\end{abstract}

\section{Introduction}

Studying equilibrium between boron species dissolved in seawater is of high geochemical importance, due to its contribution to the alkalinity and buffering capacity of seawater and thus its ability to mitigate $\mathrm{pH}$ changes, which is of vital importance for marine ecosystems [1,2].

Additionally, the equilibrium of boron species in seawater also plays a key role in studying and reconstruction of ancient seawater $\mathrm{pH}$ and atmospheric $\mathrm{pCO}_{2}$ via analysis of boron isotopic ratios in 
marine shell fossils [3-7]. To that end, an accurately determined equilibrium constant of the following isotope exchange reaction between aqueous boric acid and borate $\left(\mathrm{k}_{3-4}\right)$ :

$$
{ }^{10} \mathrm{~B}(\mathrm{OH})_{3}+{ }^{11} \mathrm{~B}(\mathrm{OH})_{4}^{-} \leftrightarrows{ }^{11} \mathrm{~B}(\mathrm{OH})_{3}+{ }^{10} \mathrm{~B}(\mathrm{OH})_{4}^{-}
$$

is of central importance and has been the subject of numerous studies. As the other more recent applications of studying the equilibrium between boron isotopes in water, we can refer to identification of nitrate pollution sources [8] and elucidation of shale weathering in the critical zone [9].

The earliest evaluation of $\mathrm{k}_{3-4}$ was theoretically calculated by Kakihana et al. [10] who reported a value of 1.0194 for this equilibrium constant. They obtained this value through the partition functions they had computed based on an empirical valence force field. This initially reported value, however, has been widely criticized in several more recent studies which suggest larger values for $\mathrm{k}_{3-4}$ ranging from 1.025 to $1.035[11-15,16]$. It can specially be important because even the slight deviation between the initially reported estimation of $\mathrm{k}_{3-4}$ and the more recent values can have a significant geochemical importance, as it results in a value for the estimated $\mathrm{pH}$ of the ocean that is almost one unit larger [16].

Among the recent evaluations of $\mathrm{k}_{3-4}$, only one work reports experimentally determined values of $1.0308 \pm 0.0023$ and $1.0272 \pm 0.0003$ obtained for pure and seawater, respectively [15]. Considering that experimental measurement of isotope fractionation in isotope exchange reactions are typically highly sensitive to experimentation, theoretical methods are more commonly employed for this purpose and are required not only to evaluate isotope fractionation but also to support experimentally determined results.

Despite the importance of precise determination of $\mathrm{k}_{3-4}$, our careful review of the theoretical works previously carried out for this purpose reveals a number of significant limitations in those works which might affect the accuracy of those reported results.

First and foremost, the employed theoretical approach for evaluation of $\mathrm{k}_{3-4}$ in all of the previously reported results are mainly limited to Normal Mode vibrational frequency Analysis (NMA) based on the harmonic oscillator approximation and thus neglect anharmonicity effects. The anharmonicity can play a significant role in isotope exchange reactions specially in light elements like boron [17].

The other limiting factor is the expected inaccuracy inherent in the employed theoretical levels in the previously reported results. The highest levels of theory employed in the previous studies are mainly limited to HF/6-31G(d) and B3LYP/6-31G(d) [13], HF/6-31G* followed by empirical scaling of frequencies [14] and PBE96 [18], which all are typically not considered as high accuracy methods for thermochemistry studies via vibrational frequency analysis [19,20]. Rustad and co-workers reported a calculation of $k_{3-4}$ at the MP2/aug-cc-pVTZ level of theory [16] which was the highest employed level of theory for this purpose so far. However, these computations were carried out for clusters with only 11 water molecules, yielding $\mathrm{k}_{3-4}$ equal to 1.033 . To empirically correct the inefficiency resulted by the small size of their studied clusters, they reported extrapolated values 
according to the pattern they observed for lower-level computations, which resulted an estimation of $\mathrm{k}_{3-4}$ between 1.026 to 1.028 .

Note that both the applied level of theory and the cluster size can significantly impact the theoretically evaluated $\mathrm{k}_{3-4}$, as shown by Rustad et al. [16]. It implies that the small size of the studied clusters is another shortcoming in the previously reported estimations of $\mathrm{k}_{3-4}$. The largest cluster size studied so far includes 34 water molecules [14], which is obviously bellow an optimal size to properly include long range interactions.

Another limitation of the studies carried out previously is the low number of configurations for calculating $\mathrm{k}_{3-4}$. As for larger molecules containing many atoms, typically several local minima exists. Consequently, geometry optimizations required for the NMA can yield quite diverse geometries with the possibility of very different energies, properties and solute-solvent interactions for each one. This necessitates employing multiple molecular configurations for the studied clusters. The work of Rustad and co-workers [16] used 10 different solute-solvent configurations, but the results reported in other studies are based on only one configuration.

Finally, the currently reported theoretical studies of $\mathrm{k}_{3-4}$ are only limited to calculated equilibrium constants in pure water, while the practical applications of equilibrium of boron isotopes mainly require estimations of $\mathrm{k}_{3-4}$ for saline water.

The main aim of the present study is to address the abovementioned limitations and provide a reliable and highly precise theoretical estimation of $\mathrm{k}_{3-4}$ for both pure and saline water. To that end, we employ vibrational frequency NMA for 60 configurations of large clusters of boric acid and borate dissolved in pure water employing a high level of theory. Nevertheless, NMA with the explicit solvent approach cannot be conveniently employed for studying multicomponent solvents since achieving appropriate sampling of a canonical ensemble in this way is challenging. Consequently, theoretical evaluation of $k_{3-4}$ in saline water is achieved via NMA with the implicit solvent approach [21] as well as path integral molecular dynamics [22,23]. The latter method also allows studying the anharmonicity impacts on the calculated partition functions.

\section{Theory}

\section{Evaluation of equilibrium constants via NMA}

The equilibrium constants of chemical reactions can be theoretically calculated via:

$$
K_{e q}=\exp \left(-\frac{\Delta A}{k_{B} T}\right)
$$

in which $\Delta A$ is the free energy change of the reaction, $k_{B}$ is the Boltzmann constant and $T$ is the temperature. Using the statistical thermodynamics definition of free energy which relates it to the partition function $(Q)$ via $A=-k_{B} T \ln (Q)$, we can rewrite 1 as: 
Table 1 The partition functions obtained based on rigid rotor harmonic oscillator approximation.

$$
\begin{aligned}
& Q_{\text {trans. }}=\left(\frac{2 \pi M k_{B} T}{h^{2}}\right)^{\frac{3}{2}} V \\
& Q_{\text {rot. }}=\frac{\sqrt{\pi}}{s}\left(\frac{8 \pi^{2} I_{A} k_{B} T}{h^{2}}\right)^{\frac{1}{2}}\left(\frac{8 \pi^{2} I_{B} k_{B} T}{h^{2}}\right)^{\frac{1}{2}}\left(\frac{8 \pi^{2} I_{C} k_{B} T}{h^{2}}\right)^{\frac{1}{2}} \quad \text { (nonlinear molecule) } \\
& Q_{\text {rot. }}=\frac{8 \pi^{2} I k_{B} T}{s h^{2}} \quad(\text { linear molecule) } \\
& Q_{\text {vib. }}=\frac{\exp \left(-\frac{h c v_{i}}{2 k_{B} T}\right)}{1-\exp \left(-\frac{h c v_{i}}{k_{B} T}\right)}
\end{aligned}
$$

$M$ is the mass of the molecule, $V$ is the volume of the system, $h$ is the Planck constant, $s$ is the symmetry number, $I$ is the principial moment of inertia, $c$ is the light speed $\left(\mathrm{cm} \mathrm{s}^{-1}\right)$ and $v_{i}$ is the $i$ 'th normal mode harmonic vibrational frequency $\left(\mathrm{cm}^{-1}\right)$.

$$
K_{e q}=\frac{\prod_{i} Q_{\text {product }, i}}{\prod_{i} Q_{\text {reactant }, i}}
$$

The total energy for each one of the reactants and products is traditionally split into contributions from translation, rotation, vibration and electronic energy, resulting in [24]:

$$
Q=\sum \exp \left(-\frac{\varepsilon_{\text {trans. }}+\varepsilon_{\text {rot. }}+\varepsilon_{\text {vib. }}+\varepsilon_{\text {elect. }}}{k_{B} T}\right)=Q_{\text {trans. }} Q_{\text {rot. }} Q_{\text {vib. }} Q_{\text {elect. }} .
$$

For isotope exchange reactions, the electronic partition functions of isotopomers cancel out as they are electronically the same and differ only by their nuclear masses. As a result, one only needs to compute the translational, rotational and vibrational partition functions which are conventionally obtained based on the Rigid Rotor Harmonic Oscillator (RRHO) approximation reported in table ?? [24], which for a nonlinear molecule results in:

$$
Q=\alpha M^{\frac{3}{2}} \frac{I_{A} I_{B} I_{C}}{s} \prod_{i} \frac{\exp \left(-\frac{h c v_{i}}{2 k_{B} T}\right)}{1-\exp \left(-\frac{-h c v_{i}}{k_{B} T}\right)} .
$$

Here $\alpha$ is a constant which depends only on the volume and temperature of the system and cancels out for reactions under constant volume and temperature.

For an isotope exchange reaction $* \mathrm{~A}+\mathrm{B} \leftrightarrows \mathrm{A}+* \mathrm{~B}$ (the heavier isotopomer is specified with *), eq. (4) can be further simplified using the Teller-Redlich product rule, which for two isotopomers 1 and 2 implies:

$$
\prod_{i} \frac{v_{i, 2}}{v_{i, 1}}=\left(\frac{M_{2}}{M_{1}}\right)^{\frac{3}{2}}\left(\frac{I_{A, 2} I_{B, 2} I_{C, 2}}{I_{A, 1} I_{B, 1} I_{C, 1}}\right)^{\frac{1}{2}} \prod_{j}\left(\frac{m_{1, j}}{m_{2, j}}\right)^{\frac{3}{2}}
$$


where $m_{i, j}$ is the mass of $j$ th atom in the isotopomer $i$. In what follows, for an isotope exchange reaction, combining (4) and (5) yields:

$$
K_{A-B}=\frac{R P F R_{B}}{R P F R_{A}},
$$

where $R P F R$ is known as the reduced partition function ratio of the solute and is calculated via:

$$
R P F R=\prod_{i} \frac{v_{i}, *}{v_{i}}\left(\frac{1-\exp \left(-\frac{h c v_{i}}{k_{b} T}\right)}{1-\exp \left(-\frac{h v_{i, *}}{k_{B} T}\right)}\right) .
$$

Calculation of equilibrium constants of isotope exchange reactions via RPFR was first proposed by Bigeleisen and Mayer [25] and has been widely used to study isotope exchange reactions [26-33]. The main advantage of the Bigeleisen and Mayer approach is that it allows calculating the isotope exchange equilibrium constants solely via normal mode vibrational frequencies. Considering that those vibrational frequencies can be obtained also experimentally and therefore without requiring calculations of principal moments of inertia for the molecules, the Bigeleisen and Mayer approach provides a practical way to evaluate the required equilibrium constants.

In the present study, in addition to the original Bigeleisen and Mayer approach, we also introduce and investigate a revision in the original implementation of the Bigeleisen and Mayer approach in the following which allows achieving a more rigorous integration of the results obtained for the multiple molecular configurations.

\section{Cost effective NMA with explicit solvent via partial normal mode analysis}

Normal mode vibrational frequencies required by NMA are conventionally computed based on the RRHO approximation. To that end, the first derivative of the system Hessian with respect to molecular geometry, i.e., the net force on each atom, must be zero, which is fulfilled by geometry optimization. For NMA in solution, taking into account the solvent effects is commonly achieved via two ways, namely implicit and explicit solvent approaches [21]. While in the explicit solvent approach, the solute is placed in the cluster of a number of solvent molecules, in the implicit solvent approach the solute is placed in the cavity of an implicitly defined solvent, instead.

For NMA with explicit solvent, the required geometry optimization is commonly applied for the whole solute-solvent cluster. This, however, makes the computations far more challenging and for high theoretical levels or large cluster sizes sometimes too expensive to be affordable. This is the reason of commonly limiting those computations to a very small number of solute-solvent configurations, small cluster sizes or low computational levels, as discussed earlier. More importantly, geometry optimization of the whole cluster can result in optimized geometries with an unpredictable density, sometimes inconsistent with that of the real studied system.

To overcome all these limitations, we introduce here the partial normal mode analysis. According to this approach, for multiple molecular configurations of solute and solvent clusters with a constant 
density, we carry out geometry optimization and NMA only for the solute while the solvent molecules are kept frozen during the optimization and NMA. Employing partial normal mode analysis followed by the thermodynamically rigorous integration, introduced in the next section, allows us to not only reduce the computational costs and challenges by several orders of magnitude but also to achieve accurate results due to studying clusters with a correct density and being able to study contributions from a higher number of configurations and for larger cluster sizes. Partial normal mode analysis for multiple molecular configurations also allows capturing anharmonic effects in the obtained results due to carrying out the NMA for diverse computed frequencies and configurations.

\section{Integrating multiple configuration results}

As discussed earlier, appropriate evaluation of thermochemistry in solution with explicit solvents requires considering contributions from multiple solute-solvent configurations. The results obtained for each configuration then should be integrated to yield the required quantities. To that end, the most convenient integration approach is obviously averaging the results obtained for all configurations. It has been the method of choice to integrate the results of 10 configurations by Rustad and co-workers [16]. For extremely large numbers of appropriately sampled configurations, this averaging yields the exact estimation of the thermodynamic properties. Nevertheless, integrating smaller numbers of configurations requires going beyond the simple arithmetic mean and employing a more rigorous integration. For this purpose, here we propose a revision of the Bigeleisen and Mayer approach which allows exploiting Boltzmann-weighted averaging, as introduced in the following.

Considering the probability of existence of any observable in the canonical ensemble, which is proportional to $e^{-\frac{H}{k_{B} T}}$ based on the Boltzmann statistics where $H$ is the Hamiltonian of the molecule [34], we suggest a more rigorous Boltzmann weighted averaging defined as:

$$
K_{e q}=\frac{\prod_{i}\left\langle Q_{\text {product }, i}\right\rangle}{\prod_{i}\left\langle Q_{\text {reactant }, i}\right\rangle},
$$

where $\langle Q\rangle$ is an ensemble-averaged partition function and is computed via:

$$
\langle Q\rangle=\frac{\sum_{i} Q_{i} \exp \left(-\frac{\varepsilon_{i}}{k_{B} T}\right)}{\sum_{i} \exp \left(-\frac{\varepsilon_{i}}{k_{B} T}\right)} .
$$

Here, $Q_{i}$ and $\varepsilon_{i}$ are the calculated partition function and energy of the solute for configuration $i$.

To implement of the Boltzmann-weighted averaging in an approach similar to the Bigeleisen and Mayer method, we propose fractionizing the Teller-Redlich product rule defined in eq. (5) for isotopomer $a$ as follows:

$$
\prod_{i} v_{i, a}=\alpha^{\prime}\left(M_{a}\right)^{\frac{3}{2}}\left(I_{A, a} I_{B, a} I_{C, a}\right)^{\frac{1}{2}} \prod_{j}\left(\frac{1}{m_{a, j}}\right)^{\frac{3}{2}},
$$


where $\alpha^{\prime}$ is a coefficient which is constant for isotopomers of a molecule. If we substitute eq. (10) in eq. (4), we obtain:

$$
Q=\frac{\alpha}{\alpha^{\prime} s} \prod_{j}\left(m_{a, j}\right)^{\frac{3}{2}} \prod_{i} v_{i} \frac{\exp \left(-\frac{h c v_{i}}{2 k_{B} T}\right)}{1-\exp \left(-\frac{h c v_{i}}{k_{B} T}\right)} .
$$

Knowing that in calculating equilibrium constants for isotope exchange reactions, the factor $\frac{\alpha}{\alpha^{\prime}} \prod_{j}\left(m_{a, j}\right)^{\frac{3}{2}}$ will be cancelled out, we can now define the Reduced Partition Function (RPF) as:

$$
\mathrm{RPF}=\frac{\alpha^{\prime} Q}{\alpha \prod_{j}\left(m_{a, j}\right)^{\frac{3}{2}}}=\frac{1}{2} \prod_{i} v_{i} \frac{\exp \left(-\frac{h c v_{i}}{2 k_{B} T}\right)}{1-\exp \left(-\frac{h c v_{i}}{k_{B} T}\right)} .
$$

Using RPF, the equilibrium constant is then calculated via:

$$
K_{A-B}=\frac{\prod_{i}\left\langle\mathrm{RPF}_{\text {product }, i}\right\rangle}{\prod_{i}\left\langle\mathrm{RPF}_{\text {reactants }, i}\right\rangle} .
$$

Here, $\langle R P F\rangle$ is the ensemble average of the reduced partition function and is calculated via $R P F_{i}$ and $\varepsilon_{i}$ which are the RPF and energy of the solute in configuration $i$ as follows:

$$
\langle\mathrm{RPF}\rangle=\frac{\sum_{i} \mathrm{RPF}_{i} \exp \left(-\frac{\varepsilon_{i}}{k_{B} T}\right)}{\sum_{i} \exp \left(-\frac{\varepsilon_{i}}{k_{B} T}\right)} .
$$

The energy of solute molecules required by eq. ((14)) can be obtained most straightforwardly using the implicit solvent approaches. To that end, we employed the IEF-PCM continuum solvation model for the same level of theory to evaluate the in-solution energy of various conformers of the solute obtained by geometry optimization in each cluster.

Considering that the $\varepsilon_{i}$ values are total ground state electronic energies and thus large negative numbers, to avoid numerical blow-up we employed subtracting the minimum of obtained $\varepsilon_{i}$ values from all of them. This is equivalent to multiply both the denominator and numerator of eq. (14) by $e^{-\frac{\min \left(\varepsilon_{i}\right)}{k_{B} T}}$ and therefore allows numerical evaluation of the reduced partition functions without losing accuracy.

\section{NMA with implicit solvent}

Although the NMA with explicit solvent possesses a number of obvious advantageous such as taking into account intricate solute-solvent interactions such as hydrogen bonding, at the same time it suffers from some limitations. In addition to challenges discussed in the previous section, the computational costs even for the partial normal mode analysis can still be high especially for large clusters and high levels of theory. The other main limitation of NMA with explicit solvent is the appropriate treatment of multi-component solvents like saline water and challenges of correctly positioning the ions. 
To overcome such limitations, for evaluation of $\mathrm{k}_{3-4}$ in saline water, we exploited NMA with implicitly defined solvent as another widely applied method to take into account solvent effects in computational chemistry [21]. A schematic illustration of implicitly defined solvents for boric acid and borate is demonstrated in figure 1.

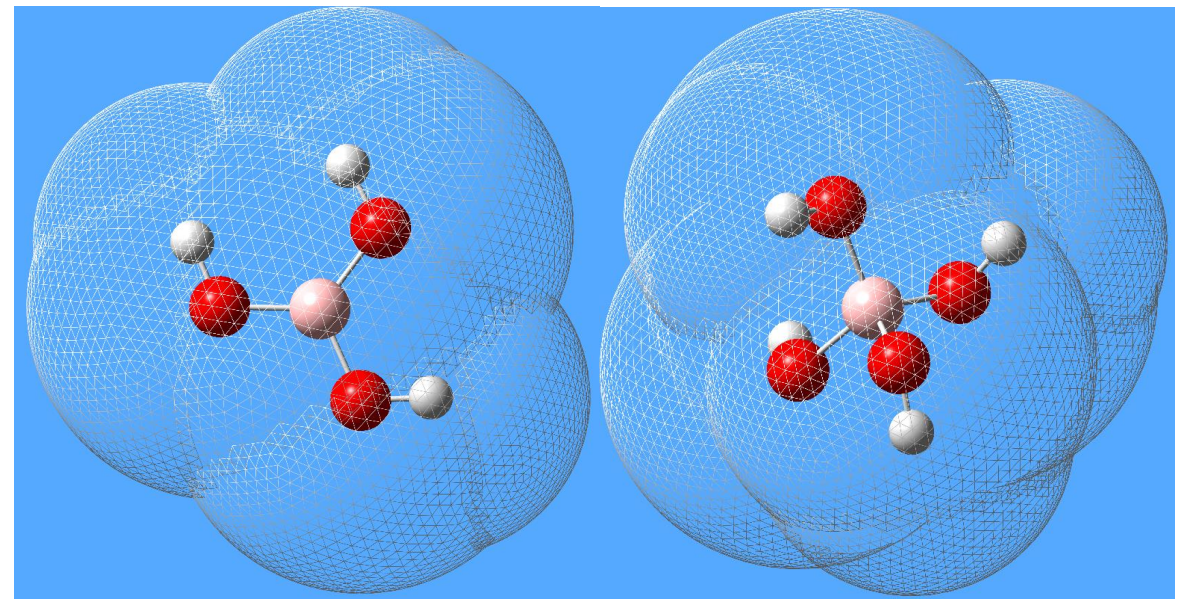

Fig. 1 Illustration of implicitly defined solvents in boric acid and borate.

Computation of $\mathrm{k}_{3-4}$ in pure water has already been reported by Liu and Tossel [14]. However, the IEF-PCM and CPCM continuum solvation models they employed at the HF/6-31G* level of theory for this purpose are commonly found to be less accurate compared to more recent solvation models like the SMx family of methods and higher levels of theory, as we have shown in a recent study [21]. Therefore, in the present study, in addition to IEF-PCM and CPCM, we also study the SMD solvation model for a high level of theory for pure and saline water.

We also employ the implicit solvent approach for evaluating the suitability of our selected cluster sizes for appropriately taking into account the long-range interactions for the NMA with explicit solvent. To that end, in addition to 60 clusters studied in vacuo, we also studied 20 clusters placed inside a cavity of implicitly defined solvents as implemented in several works [35-39].

\section{Theoretical evaluation of equilibrium constant via path integral molecu- lar dynamics}

In addition to NMA which has been the earliest theoretical approach for evaluation of equilibrium constant of isotope exchange reactions, another rigorous approach which has been widely used for this purpose is the thermodynamic integration of kinetic energies associated with isotope effects via Path Integral Molecular Dynamics (PIMD) [17,29,40-43]. Based on this approach, the equilibrium constant of an isotope exchange reaction is calculated as:

$$
K_{e q}=-\frac{3}{2} \ln \left(\frac{m_{2}}{m_{1}}\right)+\frac{1}{k_{B} T} \int_{m_{1}}^{m_{2}} \frac{\left\langle K_{E}(m)\right\rangle}{m} d m,
$$


where $m_{i}$ is the mass of isotope $i$ and $K_{E}$ is the kinetic energy. To evaluate the equilibrium constant via eq. (15), the ensemble average of kinetic energies for a number of masses alchemically transformed between $m_{1}$ and $m_{2}$ should be computed via PIMD to allow evaluating area under the $\frac{\left\langle K_{E}(m)\right\rangle}{m}$ versus $m$ curve, as required by eq. (15). Although alchemically transforming the isotope masses can be done arbitrarily, doing this via the following switching function:

$$
m(\lambda)=\frac{m_{1} m_{2}}{\left(\lambda \sqrt{m_{1}}+(1-\lambda) m_{2}\right)^{2}},
$$

where $\lambda$ is changed from 0 to 1 to yield transformed masses between $m_{1}$ and $m_{2}$, has shown to be advantageous [44] and will be employed in the present study. One common way to evaluate the kinetic energies required by eq. (15) via PIMD is based on the primitive kinetic energy estimator defined as:

$$
\left\langle K_{E}(m)\right\rangle^{\text {(primitive) }}=\frac{3 P k_{B} T}{2}-\left\langle\sum_{i=1}^{P} \frac{1}{2} m \omega_{p}^{2}\left(r_{i}-r_{i-1}\right)^{2}\right\rangle,
$$

in which $P$ is the number of beads, $\omega_{p}=\sqrt{P k_{B} T / \hbar}$ is the ring polymer frequency and $r_{i}$ is the position of the $i$ th bead of the studied isotope. However, due to the well-known drawback of the primitive estimator of kinetic energy which is its increasing variance for increasing the number of beads [45], the virial estimator of kinetic energy defined as:

$$
\left\langle k_{E}(m)\right\rangle^{(\text {virial })}=\frac{3 k_{B} T}{2}+\left\langle\frac{1}{2 P} \sum_{i=1}^{P}\left(r_{i}-r_{c}\right) \frac{\partial U}{\partial r_{i}}\right\rangle,
$$

is more commonly used for this purpose. Here, $r_{c}$ is the position of the centroid of the beads and $\frac{\partial U}{\partial r_{i}}$ is the net force on the $i$ th bead due the interaction of the atoms in the same replica and without the inter-bead interactions included.

In the present study, in evaluation of kinetic energy we exploit the features of the both abovementioned estimators to improve numerical evaluation of the integral in eq. (15). Accordingly, considering that the primitive estimator implies a linear dependency between the kinetic energies and switched masses, we fit a linear curve of the form $\left\langle K_{E}(m)\right\rangle=a+b m$, where $a$ and $b$ are constants and the kinetic energies are calculated based on the virial estimator. It allows us to reduce the numerical noises of each mass using all kinetic energy data for all other masses and also be able to solve the integral in eq. (15) analytically.

Compared to NMA, thermodynamic integration through PIMD offers a number of advantageous such as taking into account the anharmonicity effects as well as appropriate sampling of canonical ensemble and possibility of studying multi-component solvents. As a result, we employed this method for evaluation of $\mathrm{k}_{3-4}$ for the boron isotope fractionation for both pure and saline water. A schematic illustration of ring-polymers of boric acid with three beads is depicted in figure 2 


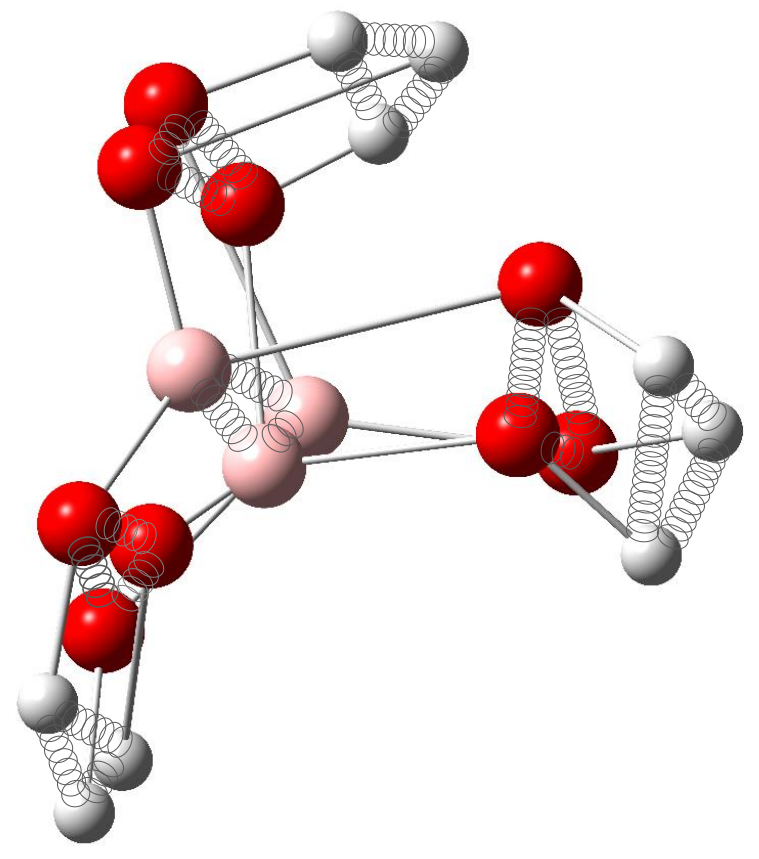

Fig. 2 Schematic illustration of a path-integral ring polymer of boric acid with three beads. The $\mathrm{B}, \mathrm{O}$ and $\mathrm{H}$ atoms are drawn in pink, red and gray color, respectively.

\section{Computational details}

\section{Evaluation of $\mathbf{k}_{3-4}$ via NMA}

To calculate $\mathrm{k}_{3-4}$ based on NMA with explicit solvent, 60 randomly selected clusters of 64 water molecules with one boric acid or borate ion in the center of the cluster were extracted from a trajectory of configurations produced by Molecular Dynamics (MD) simulations. These MD simulations were carried out for a simulation box containing 1200 water and one boric acid or borate molecule under NVT condition. The box size was fine-tuned to yield a density equal to $0.995 \mathrm{~g} / \mathrm{mol}$ which was close to the density of water at standard conditions. The inter-atomic interactions in the MD simulations were evaluated using the GFN2-xTB tight-binding method [46] and the temperature was controlled using a Nose-Hoover Chain thermostat with three chains and 50fs relaxation time. We ran the MD simulations in CP2K [47] for 10ns after 1ns equilibration with $0.5 \mathrm{fs}$ time step and took snapshots every 100 ps to generate the required configurations via screening the solute and 64 water molecules closest to it.

For the generated configurations, the normal modes were computed based on the partial normal mode analysis. To that end, the geometries of the solutes were relaxed while the solvent geometries were frozen. Considering that the employed level of theory plays a significant role in the accuracy 
of evaluated $\mathrm{k}_{3-4}$ as discussed earlier, we employed the DSD-PBEP86/QZVPP level of theory for optimization and calculations of normal modes of the solute as a rigorous and accurate method for evaluating thermodynamics quantities $[19,20,48]$ and scaled the vibrational frequencies by the recommended scaling factor of 0.9971 for improving the accuracy of this method [49]. However, for the solvent molecules, for which the normal mode calculations are not required and thus employing a high level of theory loses its importance, we used the B3LYP/6-311++G(2d,p) level of theory and computed the total energy of the cluster via the ONIOM approach.

Although clusters with 64 water molecules seem to be large enough to yield a proper solvation of the studied solutes, to further verify it, for 20 of the studied configurations we also computed the normal modes of those clusters placed in the cavity of a continuously defined solvent based on the IEF-PCM continuum solvation model.

For the computation of $\mathrm{k}_{3-4}$ solely with the implicit solvent approach, we calculated the normal modes using the DSD-PBEP86/QZVPP level of theory and the SMD, CPCM and IEF-PCM continuum solvation models for both pure and saline water. For the CPCM solvation model, in addition to the original implementation of this model in Gaussian 16, we also studied scaling the dielectric constant of the solvent via:

$$
\widetilde{\varepsilon}(\varepsilon, x)=\frac{\varepsilon+x}{x+1}
$$

for $\mathrm{x}=0.5$, as this was found to be more efficient in improving the accuracy of the CPCM method $[21,50]$.

For calculation of $\mathrm{k}_{3-4}$ in saline water, the dielectric constant of the saline water required by the continuum solvation models with the value of 70.35 recommended by Lang et al. [51] was employed. All the computations were carried out using the Gaussian 16 software [52].

\section{Evaluation of $\mathbf{k}_{3-4}$ via PIMD}

To study the anharmonic effects as well as the contributions from the configurational entropies, we employed PIMD based on the guidelines discussed earlier. To that end, we studied PIMD with 4, 8, 16, 32 and 64 beads. Using switching constants $\lambda$ linearly distributed between zero and one, 9 isotopic masses of boron varying between 10.01294 to 11.009305 were assigned via the switching function defined in eq. (16). The kinetic energies evaluated based on the virial estimator for the studied masses were then used to calculate $\mathrm{k}_{3-4}$ as described earlier.

The PIMD simulations were carried out in $\mathrm{CP} 2 \mathrm{~K}$ within the staging approach [53] for a duration of 500ps, $0.5 \mathrm{fs}$ time step, temperature controlled via Nose-Hoover Chain thermostat with three chains, periodic boundary condition and the interactions evaluated via GFN2-xTB tight-binding method [46].

For both pure and saline water, the PIMD simulation were carried out in canonical ensemble and the box size was set to yield $0.995 \mathrm{gr} / \mathrm{mol}$ density for the system. For the pure water, the simulation box contained 64 water molecule and one boric acid or borate. However, for the saline water, to set a 
reasonable molar ratio for water and $\mathrm{NaCl}$ as recommended by Zeron et al. [54], we constructed a simulation box containing one boric acid or borate, $5 \mathrm{NaCl}$ and 572 water molecules.

\section{Results and discussion}

The RPFs of boric acid and borate isotopomers calculated for 60 configurations based on the NMA with explicit solvent and the energy of the solute in each one evaluated by the IEF-PCM solvation model are reported in table 2 . The calculated $\mathrm{k}_{3-4}$ values using different integrations of these data are reported in table ??. The results obtained via NMA with explicit solvent approach and Boltzmann weighted integration show the best agreement with the upper limit of the extrapolated results reported by Rustad et al. $\left(\mathrm{k}_{3-4}=1.028\right)$ for the same approach and the highest level of theory they employed [16]. Nevertheless, all these results are slightly lower than the experimental value of $1.0308 \pm 0.0023$ reported by Klochko et al. [15].

Note that although we report integrated results for 60 configurations, the arithmetic averaging and the Boltzmann-weighted averaging result in $\mathrm{k}_{3-4}$ values of 1.0263 and 1.0279 , respectively. These two numbers still show a deviation similar to the one between upper and lower limit of the extrapolated results reported by Rustad et al. [16]. However, the results obtained via the Boltzmann weighted averaging are in much better agreement with those we obtained via NMA and the implicit solvent approach as well as PIMD reported in the following, and are almost within the uncertainty of the experimentally determined values of $1.0308 \pm 0.0023$ reported by Klochko et al. [15]. This confirms the robustness of the Boltzmann weighted averaging.

As discussed earlier, for 20 of the studied configurations, we also repeated the optimization and NMA for the same clusters but placed in the cavity of an implicitly defined solvent. However, our results showed almost exactly the same values up to 3 significant figures between the two approaches which implies that the explicit cluster size is large enough to appropriately solvate the studied solutes.

The evaluated RPFs and $\mathrm{k}_{3-4}$ calculated via NMA with implicit solvent are reported in table ??. These results show an excellent agreement with those obtained via PIMD. These results also imply the possibility of obtaining accurate results for evaluation of isotope fractionation through the implicit solvent approach despite its much lower computational challenge and cost. However, it should be noted that all this has become possible via carefully parameterizing the employed continuum solvation models to reproduce solvation free energies of numerous solutes in water and thus empirically taking into account and correcting limitations due to anharmonicity or inefficient canonical sampling [21]. The advantage of NMA with explicit solvent is that it yields results which are obtained entirely from first principle and therefore can be more reliably employed for new solvents or solutes.

According to the results of NMA with implicit solvent, the evaluated $\mathrm{k}_{3-4}$ for pure and saline water are almost the same, similar to the results we obtained via PIMD discussed in the following. These results are also in contrast to the experimentally determined values reported by Klochko et al. [15] which suggested more diverse values for $\mathrm{k}_{3-4}$ in pure and saline water equal to $1.0308 \pm 0.0023$ 
Table 2 The RPFs and conformer energies $\left(\varepsilon_{i}\right.$, Hartree/molecule) for boric acid and borate.

\begin{tabular}{|c|c|c|c|c|c|c|c|c|c|}
\hline & RPF-B $(\mathrm{OH})_{3}$ & $\varepsilon_{i}-\mathrm{B}(\mathrm{OH})_{3}$ & RPF-B $(\mathrm{OH})_{4}^{-}$ & $\varepsilon_{i}-\mathrm{B}(\mathrm{OH})_{4}$ & & RPF-B $(\mathrm{OH})_{3}$ & $\varepsilon_{i}-\mathrm{B}(\mathrm{OH})_{3}$ & RPF-B $(\mathrm{OH})_{4}^{-}$ & $\varepsilon_{i}-\mathrm{B}(\mathrm{OH})_{4}$ \\
\hline 1 & 1.232289 & -252.26 & 1.201585 & -328.159 & 31 & 1.233663 & -252.263 & 1.200363 & -328.162 \\
\hline 2 & 1.233101 & -252.26 & 1.200963 & -328.161 & 32 & 1.231332 & -252.26 & 1.200272 & -328.16 \\
\hline 3 & 1.233308 & -252.262 & 1.202624 & -328.159 & 33 & 1.231903 & -252.261 & 1.199513 & -328.16 \\
\hline 4 & 1.229193 & -252.258 & 1.200637 & -328.16 & 34 & 1.233421 & -252.258 & 1.199002 & -328.161 \\
\hline 5 & 1.232274 & -252.263 & 1.201156 & -328.157 & 35 & 1.231168 & -252.257 & 1.200133 & -328.159 \\
\hline 6 & 1.229627 & -252.257 & 1.201668 & -328.16 & 36 & 1.232375 & -252.257 & 1.19771 & -328.16 \\
\hline 7 & 1.234888 & -252.263 & 1.198716 & -328.161 & 37 & 1.231969 & -252.264 & 1.201591 & -328.159 \\
\hline 8 & 1.232739 & -252.261 & 1.203835 & -328.157 & 38 & 1.231112 & -252.261 & 1.202307 & -328.16 \\
\hline 9 & 1.233192 & -252.26 & 1.200803 & -328.161 & 39 & 1.233183 & -252.261 & 1.198171 & -328.159 \\
\hline 10 & 1.231513 & -252.261 & 1.200002 & -328.161 & 40 & 1.233118 & -252.262 & 1.198609 & -328.161 \\
\hline 11 & 1.230923 & -252.258 & 1.197027 & -328.159 & 41 & 1.23352 & -252.263 & 1.201498 & -328.158 \\
\hline 12 & 1.228097 & -252.257 & 1.196828 & -328.158 & 42 & 1.231855 & -252.261 & 1.197847 & -328.161 \\
\hline 13 & 1.232589 & -252.261 & 1.195636 & -328.16 & 43 & 1.231172 & -252.261 & 1.204232 & -328.16 \\
\hline 14 & 1.230915 & -252.259 & 1.198634 & -328.16 & 44 & 1.229711 & -252.259 & 1.198582 & -328.159 \\
\hline 15 & 1.231508 & -252.259 & 1.20012 & -328.158 & 45 & 1.232624 & -252.261 & 1.198204 & -328.162 \\
\hline 16 & 1.228483 & -252.261 & 1.201247 & -328.161 & 46 & 1.233064 & -252.263 & 1.201458 & -328.16 \\
\hline 17 & 1.23445 & -252.262 & 1.203933 & -328.159 & 47 & 1.23049 & -252.259 & 1.202082 & -328.159 \\
\hline 18 & 1.229549 & -252.256 & 1.201923 & -328.16 & 48 & 1.231707 & -252.262 & 1.203092 & -328.16 \\
\hline 19 & 1.23069 & -252.256 & 1.198874 & -328.158 & 49 & 1.232594 & -252.262 & 1.199778 & -328.161 \\
\hline 20 & 1.229597 & -252.257 & 1.196579 & -328.16 & 50 & 1.230889 & -252.26 & 1.201935 & -328.16 \\
\hline 21 & 1.231391 & -252.26 & 1.19628 & -328.16 & 51 & 1.230689 & -252.257 & 1.202002 & -328.158 \\
\hline 22 & 1.232017 & -252.261 & 1.198901 & -328.161 & 52 & 1.234509 & -252.261 & 1.196724 & -328.159 \\
\hline 23 & 1.234101 & -252.263 & 1.20088 & -328.161 & 53 & 1.230561 & -252.258 & 1.202011 & -328.16 \\
\hline 24 & 1.229961 & -252.258 & 1.204097 & -328.16 & 54 & 1.232139 & -252.26 & 1.200349 & -328.159 \\
\hline 25 & 1.231632 & -252.258 & 1.204082 & -328.159 & 55 & 1.233125 & -252.263 & 1.201442 & -328.157 \\
\hline 26 & 1.234181 & -252.261 & 1.198961 & -328.158 & 56 & 1.231232 & -252.257 & 1.203445 & -328.159 \\
\hline 27 & 1.231185 & -252.259 & 1.200437 & -328.159 & 57 & 1.229698 & -252.254 & 1.202555 & -328.16 \\
\hline 28 & 1.230308 & -252.26 & 1.19904 & -328.162 & 58 & 1.233923 & -252.261 & 1.201612 & -328.162 \\
\hline 29 & 1.231356 & -252.259 & 1.195705 & -328.16 & 59 & 1.230443 & -252.258 & 1.197761 & -328.16 \\
\hline 30 & 1.23166 & -252.26 & 1.200217 & -328.161 & 60 & 1.229274 & -252.255 & 1.195276 & -328.159 \\
\hline
\end{tabular}


Table 3 Integrated results of RPFs and the evaluated $k_{3-4}$.

\begin{tabular}{llll}
\hline & $\mathrm{B}(\mathrm{OH})_{3}$ & $\mathrm{~B}(\mathrm{OH})_{4}^{-}$ & $\mathrm{k}_{3-4}$ \\
\hline Arithmetic averaging & 1.2317 & 1.2002 & 1.0263 \\
Boltzmann weighted averaging & 1.2330 & 1.1997 & 1.0278 \\
\hline
\end{tabular}

Table 4 Calculated RPFs and the evaluated $\mathrm{k}_{3-4}$ via different continuum solvation models for pure and saline water

\begin{tabular}{lllllll}
\hline & \multicolumn{3}{c}{ Pure water } & \multicolumn{3}{c}{ Saline water } \\
\hline & $\mathrm{B}(\mathrm{OH})_{3}$ & $\mathrm{~B}(\mathrm{OH})_{4}^{-}$ & $\mathrm{k}_{3-4}$ & $\mathrm{~B}(\mathrm{OH})_{3}$ & $\mathrm{~B}(\mathrm{OH})_{4}^{-}$ & $\mathrm{k}_{3-4}$ \\
\hline $\mathrm{SMD}$ & 1.2206 & 1.182 & 1.0327 & 1.2207 & 1.182 & 1.0327 \\
$\mathrm{IEF}-\mathrm{PCM}$ & 1.2267 & 1.1902 & 1.0306 & 1.2267 & 1.1902 & 1.0306 \\
$\mathrm{CPCM}$ & 1.2266 & 1.1901 & 1.0307 & 1.2266 & 1.1901 & 1.0306 \\
$\mathrm{CPCM}(\mathrm{x}=0.5)$ & 1.2267 & 1.1902 & 1.0307 & 1.2267 & 1.1902 & 1.0307 \\
\hline
\end{tabular}

and $1.0272 \pm 0.0003$, respectively. However, considering that the uncertainty in the experimentally measured data reported by Klochko et al. for $\mathrm{k}_{3-4}$ in pure water is much higher than those observed for saline water, implying some overlapping values in between, we can conclude that $\mathrm{k}_{3-4}$ is almost the same in pure and saline water.

The evaluated $\mathrm{k}_{3-4}$ obtained via PIMD for pure and saline water for different path integral bead numbers are reported in table ??. These results show that ring polymers with 32 beads yield converged results for estimated $\mathrm{k}_{3-4}$. These results also show a good agreement with the theoretically predicted results obtained via NMA as well as the experimentally measured values. Similar to the results obtained via NMA with implicit solvent, here also the results imply that the $\mathrm{k}_{3-4}$ for both pure and saline water are not significantly different.

Table 5 Evaluated $\mathrm{k}_{3-4}$ via PIMD for pure and saline water

\begin{tabular}{lll}
\hline No. beads & Pure water & Saline water \\
\hline 4 & 1.013377 & 1.013113 \\
8 & 1.023831 & 1.02616 \\
16 & 1.031404 & 1.030177 \\
32 & 1.033596 & 1.030278 \\
64 & 1.030522 & 1.030118 \\
\hline
\end{tabular}




\section{Conclusion}

In the present study, theoretical evaluation of $\mathrm{k}_{3-4}$ using high level NMA with the explicit solvent approach and three different implicit solvent approaches as well as PIMD was studied. By comparing various high-precision theoretical methods employed in the present study with those reported elsewhere by Klochko et al. [15] via experimental techniques and theoretically reported results of Rustad et al. [16], we can propose the most probable estimation of $\mathrm{k}_{3-4}$ within 1.028 to 1.030 for both pure and saline water.

\section{Acknowledgment}

This project has received funding in the framework of European Union's Horizon 2020 research and innovation programme under the Marie Sklodowska-Curie grant agreement No 643084. The authors wish to thank Simone Knief and Karsten Balzer in the computing center of Christian-Albrechts University of Kiel for their assistance and technical supports for running the computations on the high-performance computation hardware of Kiel University. 


\section{References:}

1. Dickson, A. G.; Goyet, C. Handbook of methods for the analysis of the various parameters of the carbon dioxide system in sea water. Version 2; Oak Ridge National Lab., TN (United States): 1994.

2. Zeebe, R. E.; Sanyal, A.; Ortiz, J. D.; Wolf-Gladrow, D. A., A theoretical study of the kinetics of the boric acid-borate equilibrium in seawater. Marine Chemistry 2001, 73 (2), 113-124.

3. Vengosh, A.; Kolodny, Y.; Starinsky, A.; Chivas, A. R.; McCulloch, M. T., Coprecipitation and isotopic fractionation of boron in modern biogenic carbonates. Geochimica et Cosmochimica Acta 1991, 55 (10), 2901-2910.

4. Hemming, N. G.; Hanson, G. N., Boron isotopic composition and concentration in modern marine carbonates. Geochimica et Cosmochimica Acta 1992, 56 (1), 537-543.

5. Pearson, P. N.; Palmer, M. R., Atmospheric carbon dioxide concentrations over the past 60 million years. Nature 2000, 406 (6797), 695-699.

6. Tyrrell, T.; Zeebe, R. E., History of carbonate ion concentration over the last 100 million years. Geochimica et Cosmochimica Acta 2004, 68 (17), 3521-3530.

7. De La Vega, E.; Foster, G. L.; Martínez-Botí, M. A.; Anagnostou, E.; Field, M. P.; Kim, M. H.; Watson, P.; Wilson, P. A., Automation of boron chromatographic purification for $\delta 11 \mathrm{~B}$ analysis of coral aragonite. Rapid Communications in Mass Spectrometry 2020, 34 (11), e8762.

8. Carrey, R.; Ballesté, E.; Blanch, A. R.; Lucena, F.; Pons, P.; López, J. M.; Rull, M.; Solà, J.; Micola, N.; Fraile, J., Combining multi-isotopic and molecular source tracking methods to identify nitrate pollution sources in surface and groundwater. Water Research 2021, 188, 116537.

9. Noireaux, J.; Sullivan, P. L.; Gaillardet, J.; Louvat, P.; Steinhoefel, G.; Brantley, S. L., Developing boron isotopes to elucidate shale weathering in the critical zone. Chemical Geology 2021, 559, 119900.

10. Kakihana, H.; Kotaka, M.; Satoh, S.; Nomura, M.; Okamoto, M., Fundamental studies on the ion-exchange separation of boron isotopes. Bulletin of the Chemical Society of Japan 1977, 50 (1), 158-163.

11. Oi, T.; Yanase, S., Calculations of reduced partition function ratios of hydrated monoborate anion by the ab initio moleculaSr orbital theory. Journal of nuclear science and technology 2001, 38 (6), 429-432.

12. Yamahira, M.; Oi, T., Calculations of reduced partition function ratios of hydrated boric acid molecule by the ab initio molecular orbital theory. Journal of nuclear science and technology 2004, $41(8), 832-836$.

13. Zeebe, R. E., Stable boron isotope fractionation between dissolved B (OH) 3 and B (OH) 4-. Geochimica et Cosmochimica Acta 2005, 69 (11), 2753-2766.

14. Liu, Y.; Tossell, J. A., Ab initio molecular orbital calculations for boron isotope fractionations on boric acids and borates. Geochimica et Cosmochimica Acta 2005, 69 (16), 3995-4006. 
15. Klochko, K.; Kaufman, A. J.; Yao, W.; Byrne, R. H.; Tossell, J. A., Experimental measurement of boron isotope fractionation in seawater. Earth and Planetary Science Letters 2006, 248 (1-2), 276-285. 16. Rustad, J. R.; Bylaska, E. J.; Jackson, V. E.; Dixon, D. A., Calculation of boron-isotope fractionation between B (OH) 3 (aq) and B (OH) 4-(aq). Geochimica et Cosmochimica Acta 2010, 74 (10), 2843-2850.

17. Dupuis, R.; Benoit, M.; Tuckerman, M. E.; Meheut, M., Importance of a fully anharmonic treatment of equilibrium isotope fractionation properties of dissolved ionic species as evidenced by $\mathrm{Li}+$ (aq). Accounts of chemical research 2017, 50 (7), 1597-1605.

18. Rustad, J. R.; Bylaska, E. J., Ab initio calculation of isotopic fractionation in B (OH) $3(\mathrm{aq})$ and BOH4-(aq). Journal of the American Chemical Society 2007, 129 (8), 2222-2223.

19. Goerigk, L.; Hansen, A.; Bauer, C.; Ehrlich, S.; Najibi, A.; Grimme, S., A look at the density functional theory zoo with the advanced GMTKN55 database for general main group thermochemistry, kinetics and noncovalent interactions. Physical Chemistry Chemical Physics 2017, 19 (48), 3218432215.

20. Kesharwani, M. K.; Brauer, B.; Martin, J. M., Frequency and zero-point vibrational energy scale factors for double-hybrid density functionals (and other selected methods): can anharmonic force fields be avoided? The Journal of Physical Chemistry A 2014, 119 (9), 1701-1714.

21. Alibakhshi, A.; Hartke, B., Improved prediction of solvation free energies by machine-learning polarizable continuum solvation model. Nature Communications 2021, 12 (1), 1-7.

22. Steffen, J., A new class of reaction path based potential energy surfaces enabling accurate black box chemical rate constant calculations. The Journal of chemical physics 2019, 150 (15), 154105.

23. Steffen, J.; Hartke, B., Cheap but accurate calculation of chemical reaction rate constants from ab initio data, via system-specific, black-box force fields. The Journal of chemical physics 2017, 147 (16), 161701.

24. McQuarrie, D. A., Statistical thermodynamics. 1973.

25. Bigeleisen, J.; Mayer, M. G., Calculation of equilibrium constants for isotopic exchange reactions. The Journal of Chemical Physics 1947, 15 (5), 261-267.

26. Sato, A.; Bernier-Latmani, R.; Hada, M.; Abe, M., Ab initio and steady-state models for uranium isotope fractionation in multi-step biotic and abiotic reduction. Geochimica et Cosmochimica Acta 2021.

27. Pinilla, C.; De Moya, A.; Rabin, S.; Morard, G.; Roskosz, M.; Blanchard, M., First-principles investigation of equilibrium iron isotope fractionation in Fe1- xSx alloys at Earth's core formation conditions. Earth and Planetary Science Letters 2021, 569, 117059.

28. Balan, E.; Noireaux, J.; Mavromatis, V.; Saldi, G. D.; Montouillout, V.; Blanchard, M.; Pietrucci, F.; Gervais, C.; Rustad, J. R.; Schott, J., Theoretical isotopic fractionation between structural boron in carbonates and aqueous boric acid and borate ion. Geochimica et Cosmochimica Acta 2018, 222, 117-129.

29. Pinilla, C.; Blanchard, M.; Balan, E.; Natarajan, S. K.; Vuilleumier, R.; Mauri, F., Equilibrium magnesium isotope fractionation between aqueous $\mathrm{Mg} 2+$ and carbonate minerals: insights from path integral molecular dynamics. Geochimica et Cosmochimica Acta 2015, 163, 126-139.

30. Feng, C.; Qin, T.; Huang, S.; Wu, Z.; Huang, F., First-principles investigations of equilibrium calcium isotope fractionation between clinopyroxene and Ca-doped orthopyroxene. Geochimica et Cosmochimica Acta 2014, 143, 132-142.

31. Huang, F.; Chen, L.; Wu, Z.; Wang, W., First-principles calculations of equilibrium Mg isotope 
fractionations between garnet, clinopyroxene, orthopyroxene, and olivine: implications for $\mathrm{Mg}$ isotope thermometry. Earth and Planetary Science Letters 2013, 367, 61-70.

32. Javoy, M.; Balan, E.; Méheut, M.; Blanchard, M.; Lazzeri, M., First-principles investigation of equilibrium isotopic fractionation of $\mathrm{O}$-and Si-isotopes between refractory solids and gases in the solar nebula. Earth and Planetary Science Letters 2012, 319, 118-127.

33. Kowalski, P. M.; Jahn, S., Prediction of equilibrium Li isotope fractionation between minerals and aqueous solutions at high $\mathrm{P}$ and $\mathrm{T}$ : an efficient ab initio approach. Geochimica et Cosmochimica Acta 2011, 75 (20), 6112-6123.

34. Tuckerman, M., Statistical mechanics: theory and molecular simulation. Oxford university press: 2010.

35. da Silva, E. F.; Svendsen, H. F.; Merz, K. M., Explicitly representing the solvation shell in continuum solvent calculations. The Journal of Physical Chemistry A 2009, 113 (22), 6404-6409.

36. Hayes, J. M.; Bachrach, S. M., Effect of micro and bulk solvation on the mechanism of nucleophilic substitution at sulfur in disulfides. The Journal of Physical Chemistry A 2003, 107 (39), $7952-$ 7961.

37. da Silva, C. O.; Mennucci, B.; Vreven, T., Combining microsolvation and polarizable continuum studies: New insights in the rotation mechanism of amides in water. The Journal of Physical Chemistry A 2003, 107 (34), 6630-6637.

38. Fernández-Ramos, A.; Miller, J. A.; Klippenstein, S. J.; Truhlar, D. G., Modeling the kinetics of bimolecular reactions. Chemical reviews 2006, 106 (11), 4518-4584.

39. Li, Y.; Hartke, B., Assessing Solvation Effects on Chemical Reactions with Globally Optimized Solvent Clusters. ChemPhysChem 2013, 14 (12), 2678-2686.

40. Wang, L.; Ceriotti, M.; Markland, T. E., Quantum kinetic energy and isotope fractionation in aqueous ionic solutions. Physical Chemistry Chemical Physics 2020, 22 (19), 10490-10499.

41. Daengngern, R.; Kobayashi, O.; Kungwan, N.; Ngaojampa, C.; Tachikawa, M., Nuclear quantum and $\mathrm{H} / \mathrm{D}$ isotope effects on three-centered bonding diborane: Path integral molecular dynamics simulations. International Journal of Quantum Chemistry 2020, 120 (10), e26179.

42. Brela, M. Z.; Prah, A.; Boczar, M.; Stare, J.; Mavri, J., Path integral calculation of the hydrogen/deuterium kinetic isotope effect in monoamine oxidase a-catalyzed decomposition of benzylamine. Molecules 2019, 24 (23), 4359.

43. Pinilla, C.; Blanchard, M.; Balan, E.; Ferlat, G.; Vuilleumier, R.; Mauri, F., Equilibrium fractionation of $\mathrm{H}$ and $\mathrm{O}$ isotopes in water from path integral molecular dynamics. Geochimica et Cosmochimica Acta 2014, 135, 203-216.

44. Marsalek, O.; Chen, P.-Y.; Dupuis, R.; Benoit, M.; Méheut, M.; Bačić, Z.; Tuckerman, M. E., Efficient calculation of free energy differences associated with isotopic substitution using path-integral molecular dynamics. Journal of chemical theory and computation 2014, 10 (4), 1440-1453.

45. Herman, M.; Bruskin, E.; Berne, B., On path integral Monte Carlo simulations. The Journal of Chemical Physics 1982, 76 (10), 5150-5155.

46. Bannwarth, C.; Ehlert, S.; Grimme, S., GFN2-xTB-An accurate and broadly parametrized self-consistent tight-binding quantum chemical method with multipole electrostatics and densitydependent dispersion contributions. Journal of chemical theory and computation 2019, 15 (3), $1652-$ 1671.

47. Hutter, J.; Iannuzzi, M.; Schiffmann, F.; VandeVondele, J., cp2k: atomistic simulations of condensed matter systems. Wiley Interdisciplinary Reviews: Computational Molecular Science 2014, 4 
(1), 15-25.

48. Alibakhshi, A., High precision evaluation of the combustion enthalpy by ab-intio computations. ChemRxiv. Cambridge: Cambridge Open Engage 2021.

49. Kesharwani, M. K.; Sylvetsky, N.; Martin, J. M. In Surprising performance for vibrational frequencies of the distinguishable clusters with singles and doubles (DCSD) and MP2. 5 approximations, AIP Conference Proceedings, AIP Publishing: 2017; p 030005.

50. Klamt, A.; Moya, C.; Palomar, J., A comprehensive comparison of the IEFPCM and SS (V) PE continuum solvation methods with the COSMO approach. Journal of chemical theory and computation 2015, 11 (9), 4220-4225.

51. Lang, R.; Zhou, Y.; Utku, C.; Le Vine, D., Accurate measurements of the dielectric constant of seawater at $\mathrm{L}$ band. Radio Science 2016, 51 (1), 2-24.

52. Frisch, M.; Trucks, G.; Schlegel, H.; Scuseria, G.; Robb, M.; Cheeseman, J.; Scalmani, G.; Barone, V.; Petersson, G.; Nakatsuji, H., Gaussian 16. Gaussian, Inc. Wallingford, CT: 2016.

53. Tuckerman, M.; Berne, B., Vibrational relaxation in simple fluids: Comparison of theory and simulation. The Journal of chemical physics 1993, 98 (9), 7301-7318.

54. Zeron, I. M.; Gonzalez, M. A.; Errani, E.; Vega, C.; Abascal, J. L., “In Silico” Seawater. Journal of Chemical Theory and Computation 2021, 17 (3), 1715-1725. 
